\title{
Cuidado, HIV/Aids e atenção primária no Brasil: desafio para a atenção no Sistema Único de Saúde?
}

\author{
Eduardo Alves Melo, ${ }^{1}$ Ivia Maksud ${ }^{2}$ e Rafael Agostini ${ }^{2}$
}

Como citar Melo EA, Maksud I, Agostini R. Cuidado, HIV/Aids e atenção primária no Brasil: desafio para a atenção no Sistema Único de Saúde? Rev Panam Salud Publica. 2018;42:e151. https:/ /doi.org/10.26633/ RPSP.2018.151

RESUMO

No Brasil, o Sistema Único de Saúde (SUS) prevê a organização de uma rede hierarquizada e regionalizada de serviços de saúde tendo a atenção primária à saúde (APS) como ordenadora e porta de entrada para os serviços. Recentemente, novas diretrizes e experiências brasileiras designaram para a APS um papel de maior protagonismo no tema das politicas de HIV/Aids, que até então desenvolviam-se, no seu componente assistencial, centralmente em serviços especializados. Este artigo contextualiza e problematiza esse recente processo de descentralização do cuidado às pessoas vivendo com HIV/Aids no SUS. A partir de 2011, novas tecnologias diagnósticas (como os testes rápidos) foram implantadas na APS no Brasil, ampliando o acesso à testagem e promovendo um aumento do número de diagnósticos de HIV na APS. A partir de 2013, diretrizes e recomendações incentivaram também o acompanhamento de pessoas com HIV/Aids no âmbito da APS. Nesse contexto, o presente artigo examina a relação entre APS e atenção especializada, as questões de acesso, estigma e confidencialidade na APS e o modo de organização e funcionamento das equipes de saúde da família, notadamente a vinculação formal entre moradores a equipes. Conclui-se que o enfrentamento de vários desafios - de ordem moral, ética, técnica, organizacional e política - é necessário para ampliar as possibilidades de acesso e a qualidade do cuidado na APS para as pessoas vivendo com HIV/Aids no Brasil.

Palavras-chave Atenção primária à saúde; síndrome de imunodeficiência adquirida; assistência integral à saúde; Brasil.

O Sistema Único de Saúde (SUS) do Brasil, criado a partir da Constituição de 1988, representa a materialização jurídico-institucional das lutas dos movimentos pela reforma sanitária e tem como princípios e diretrizes centrais a universalidade, a equidade, a integralidade, a descentralização e o controle

\footnotetext{
Fundação Oswaldo Cruz, Escola Nacional de Saúde Pública (ENSP), Rio de Janeiro (RJ), Brasil. Correspondência: eduardo.melo@ensp.fiocruz.br

2 Fundação Oswaldo Cruz, Instituto Fernandes Figueira, Rio de Janeiro (RJ), Brasil.
}

social. Prevê um sistema organizado em redes de atenção à saúde (RAS) hierarquizadas e regionalizadas (1), visando à produção de cuidados integrados e integrais e à racionalização diante dos problemas gerados pela fragmentação das ações de saúde e pelos custos em saúde $(2,3)$.

Internacionalmente, a atenção primária à saúde (APS) tem sido pensada a partir de alguns atributos, destacando-se primeiro contato, acessibilidade, longitudinalidade e coordenação do cuidado, abordagem ampliada e competência cultural (4). Esses atributos colocam, para a APS (também chamada no Brasil de atenção básica), a missão desafiadora de operar como base de ordenamento e porta de entrada preferencial das RAS no SUS, com alta resolutividade (clínica e sanitária) e integração com os demais serviços que compõem as RAS.

No Brasil, o SUS apresenta avanços e inovações - em áreas como imunização, saúde mental, APS, HIV/Aids e transplantes, entre outras - que, entretanto, evidenciam uma implantação mais através 
de programas e políticas específicas e parciais do que de modo sistêmico e integrado (3). Isso se deve, ao menos em parte, a problemas como o subfinanciamento, à gestão envolvendo três entes federados autônomos (União, estados e municípios), à influência do setor privado de saúde, à profunda heterogeneidade regional do Brasil e ao contexto de implantação do SUS (desfavorável a políticas de proteção social universais), o que acaba conformando "vários SUS". As disparidades socioeconômicas e regionais se expressam também em termos de saúde e de sistema de saúde, sendo a administração de um sistema tão descentralizado um desafio complexo (5).

Recentemente, novas diretrizes nacionais e experiências locais têm colocado a APS em posição de protagonismo no tema do HIV / Aids, com papel de manter e ampliar ações de promoção, prevenção e diagnóstico e de incorporar o acompanhamento de usuários com HIV. Até então, as políticas de HIV/Aids, nas quais o Brasil tem se destacado no cenário mundial, tinham seu componente assistencial desenvolvido principalmente nos serviços especializados.

Considerando esses aspectos, o objetivo deste artigo é contextualizar o recente processo de descentralização do cuidado às pessoas com HIV / Aids para o âmbito da APS no Brasil - tema ainda escasso na literatura científica - e problematizar potencialidades e desafios como o direito das pessoas com HIV/Aids a uma atenção integral (considerando necessidades de saúde, acesso a ações e serviços bem como direitos humanos), por um lado, e a necessidade de fortalecer a APS e sua integração nas RAS, por outro. Ressaltamos aqui o entendimento de que a construção e a implantação de políticas públicas são processos sociopolíticos nos quais distintos atores e projetos/agendas se apresentam, comportando inclusive ações e reações em distintos planos e arenas, tais como o plano governamental, dos serviços de saúde e da sociedade civil.

\section{HIV NO BRASIL}

Os primeiros casos de infecção pelo HIV datam do início da década de 1980, nos Estados Unidos. No Brasil, as respostas à epidemia se iniciaram em 1982, antes da constituição do SUS, com a criação das primeiras organizações de mobilização comunitária, o programa estadual de São Paulo (região Sudeste), em 1983, e o programa nacional no Ministério da Saúde, em 1986 (6). Depois desses anos iniciais, a epidemia passou a ser caracterizada por um intenso processo de respostas colaborativas, incluindo desde pessoas vivendo com HIV / Aids (PVHA) até áreas técnicas $(7,8)$.

A história brasileira foi marcada pelo desafio de incorporar as respostas à epidemia em longo prazo. O trabalho conjunto entre diversos setores e atores passou por acordos de empréstimo junto ao Banco Mundial para financiamento das atividades de prevenção durante as duas primeiras décadas. Além disso, o Brasil inovou ao incorporar os medicamentos antirretrovirais ao SUS, contrariando recomendações do Banco Mundial. Essa medida promoveu uma alteração radical nos quadros clínicos e epidemiológicos, resultando na redução da mortalidade e no aumento da expectativa de vida das $\operatorname{PVHA}(7,8)$.

Ao longo dessa história, o modelo clínico adotado foi inicialmente de acompanhamento na atenção especializada, em geral por médicos infectologistas. Os serviços de atenção especializada (SAE), de caráter ambulatorial, estavam inseridos em policlínicas e hospitais. Os diagnósticos eram realizados nessas mesmas unidades e, posteriormente, se concentraram nos Centros de Testagem e Aconselhamento (CTA). A participação da APS nesse processo só foi intensificada depois dos anos 2000, por meio de sua responsabilização pelas ações de prevenção e aconselhamento e, recentemente, de testagem $(9,10)$. O acompanhamento das PVHA a partir de uma estratificação de riscos foi a última etapa do processo de descentralização do cuidado e a sua implantação está em curso de forma incipiente em alguns municípios.

Sendo assim, em anos mais recentes, o cenário das respostas à epidemia de $\mathrm{HIV} /$ Aids vem sendo significativamente alterado no Brasil, numa tentativa de adequação a metas globais de controle (11). Em relação à prevenção, destacam-se a incorporação dos testes rápidos no SUS e sua paulatina descentralização para a APS, a partir da Estratégia Saúde da Família (ESF), e para unidades móveis, em parceria com a sociedade civil; a ampliação e unificação das ações para a profilaxia pós-exposição (PEP); e a incorporação da profilaxia pré-exposição (PrEP) para determinadas populações no âmbito do SUS. No campo da atenção, por sua vez, destacam-se a elegibilidade de qualquer PVHA para o tratamento antirretroviral e a adoção do tratamento como prevenção; e a incorporação da dose fixa combinada como primeira linha de tratamento, com a inclusão de novos medicamentos.

De acordo com dados epidemiológicos oficiais, entre 1980 e 2017 foram notificados 882810 casos de Aids no Brasil, sendo $576245(65,3 \%)$ em homens e 306444 (34,7\%) em mulheres. Os casos estão mais concentrados nas regiões $\mathrm{Su}$ deste $(52,3 \%)$ e Sul $(20,1 \%)$, seguidas das regiões Nordeste $(15,4 \%)$, Norte $(6,1 \%)$ e Centro-Oeste (6, 0\%). De 2007 até 2017, foram notificados 194217 casos de infecção pelo HIV no Brasil. No ano de 2014, a principal via de transmissão entre indivíduos com 13 anos ou mais foi a sexual, em ambos os sexos. Entre indivíduos menores de 13 anos, a quase totalidade dos casos teve como via de infecção a transmissão vertical (12).

\section{APS NO BRASIL}

A APS é um elemento estratégico na constituição das RAS no SUS. A ESF vem sendo considerada uma estratégia de reorientação do modelo assistencial no SUS, tendo se expandido a praticamente todos os municípios e regiões brasileiras a partir de indução financeira federal feita pelo Ministério da Saúde, sendo uma expressão do processo de descentralização intensificado a partir dos anos 1990. A ESF tem maiores coberturas em territórios com populações em situação de maior vulnerabilidade social (13). Atualmente, existem cerca de 41 mil equipes de saúde da família, o que representa uma cobertura de aproximadamente 65\% dos 204 milhões de brasileiros.

A Política Nacional de Atenção Básica (PNAB) coloca, para a APS, as funções de base e porta de entrada preferencial da RAS, de ser resolutiva e de coordenar o cuidado dos usuários, adotando diretrizes como territorialização, adscrição de clientela e trabalho em equipe, entre outros, e desenvolvendo ações de promoção, prevenção, cura, manutenção da saúde, reabilitação e redução de danos, considerando pessoas e coletividades em seus contextos cultural e socioeconômico (14).

A principal forma de organização da APS no Brasil é através das equipes de saúde da família, com médico generalista, enfermeiro, técnico de enfermagem e agentes comunitários de saúde (ACS), 
podendo incluir profissionais de saúde bucal, bem como suporte de profissionais como psicólogos, nutricionistas, fisioterapeutas e psiquiatras, entre outros, conformando Núcleos de Apoio à Saúde da Família (NASF) que realizam práticas como discussão de casos, atendimentos conjuntos e ações coletivas, buscando ampliar a capacidade de cuidado da APS. Cada equipe de saúde da família é responsável por cuidar, em média, de 3000 pessoas, podendo variar para mais ou menos a depender das características do território e da população (grau de vulnerabilidade social, por exemplo). Os ACS se caracterizam por morar no mesmo bairro onde se localizam os serviços que abrigam as equipes de saúde de família e a população sob sua responsabilidade, sendo considerados mediadores entre população e serviços de APS. Os serviços da atenção básica, denominados genericamente de unidades básicas de saúde (UBS), são geridos pelos municípios, a partir de diretrizes nacionais e financiamento compartilhado entre municípios, estados e União.

As três principais linhas de atuação da APS no SUS são o acolhimento da demanda espontânea, garantindo acesso, a oferta de cuidado continuado e a atenção aos problemas/riscos coletivos em saúde (15). Para tanto, a APS é organizada de modo territorial, com alto grau de proximidade à dinâmica social e às condições e modos de vida das pessoas. Num país diverso, desigual e grande como o Brasil, há diferentes realidades de APS que coexistem com elementos comuns (16).

Assim como outros componentes do SUS, a APS enfrenta limitações e problemas, a despeito dos avanços. É importante sinalizar, ainda, que algumas práticas e ações já são incorporadas à atenção básica de maneira ampla, como aquelas voltadas à saúde da criança, ao cuidado pré-natal e ao cuidado de pessoas com diabetes e hipertensão. Por outro lado, ações no campo da saúde mental, da reabilitação e de condições como HIV / Aids, ainda que existam, são menos características da atenção básica, ou realizadas nesse espaço de modo mais parcial.

Houve uma importante inflexão na PNAB e em outras políticas nacionais dirigidas à APS no Brasil entre os anos de 2011 e 2014, com destaque para o Programa Mais Médicos (17). Por outro lado, a recente revisão normativa da PNAB, ocorrida em 2017, num cenário social, político e econômico negativo para o
Brasil e para o SUS, tem sido objeto de muitos protestos e preocupações quanto ao risco de enfraquecimento da ESF e reforço de um modelo de APS residual e anterior à ESF.

\section{O CUIDADO ÀS PESSOAS VIVENDO COM HIV/AIDS NA APS BRASILEIRA}

Entre os anos de 2011 e 2012, o Ministério da Saúde introduziu novas tecnologias diagnósticas na APS, com destaque, conforme já mencionado, para os testes rápidos (gravidez, sífilis e HIV, entre outros), ampliando o acesso à testagem e o aumento do diagnóstico de HIV na APS em todas as regiões do país. A partir de 2013, o Ministério da Saúde passou a adotar também diretrizes e recomendações de incentivo ao acompanhamento de pessoas com HIV/Aids (com quadro de baixo risco) na atenção básica dos municípios (18). Simultaneamente, alguns municípios brasileiros com grande investimento na estruturação da APS, como Curitiba e Rio de Janeiro (localizados nas regiões Sul e Sudeste, respectivamente), passaram efetivamente a descentralizar o cuidado a pessoas com HIV para a APS (18). Ainda que ações de promoção e prevenção sobre HIV já estivessem difundidas nas equipes de saúde da família, o diagnóstico de novos casos (em todas as regiões) e, principalmente, o acompanhamento de usuários com diagnóstico positivo (em alguns municípios) são processos muito recentes, cercados de polêmica e ainda pouco estudados.

Considerando as trajetórias de construção da APS no Brasil e da resposta brasileira ao HIV, formulações desenvolvidas na saúde coletiva brasileira e em outros campos de saber, bem como o contexto mais geral do SUS e do Brasil, alguns elementos requerem atenção especial. O primeiro deles é a relação entre APS e atenção especializada, considerando a centralidade do médico no acompanhamento das PVHA. Embora "compartilhado" entre os dois "níveis" de atenção, segundo a proposta programática, o cuidado que se opera pelos serviços de saúde pode se configurar como uma tensão. Na experiência brasileira, médicos que estão na APS, generalistas, podem ser vistos por médicos especialistas como "recém-formados" ou "em fim de carreira", com formação deficitária para o "manejo" da infecção, sobrecarga de trabalho e funções que não seriam atribuições de médicos, resultando num tratamento inadequado a PVHA. Seria o caso de pensar que isso configura um embate entre duas formas/lógicas de fazer medicina, ou entre duas epistemologias do cuidado, uma mais integral/ holista e outra mais biomédica (19)? Como pensar um "cuidado compartilhado" nesse caso? Proposições como a do apoio matricial, presente na experiência dos NASF, no qual especialistas e generalistas se relacionam em torno de casos concretos e realizam ações como discussão de casos e consultas conjuntas, podem ser um caminho fértil.

O segundo elemento de problematização refere-se às questões de acesso, estigma e confidencialidade na APS. Uma das justificativas para a descentralização do acompanhamento das PVHA seria a facilidade de acesso à atenção. No entanto, a literatura aponta o receio de PVHA de terem sua sorologia revelada para a comunidade a partir do compartilhamento de informações e decisões na equipe multiprofissional, característica das equipes de saúde de família (10). Em que medida a territorialidade pode, portanto, ampliar as possibilidades de cuidado ou exposição a preconceitos?

O terceiro elemento a ser destacado diz respeito ao modo de organização e funcionamento das equipes de saúde de família, notadamente a vinculação formal de moradores a equipes. Cada equipe de saúde de família é responsável por um território geográfico, com vinculação da população que nele reside ou, em alguns casos, trabalha. Existem alguns limites nessa organização. De um lado está a noção formal de vínculo, que deveria supor uma relação de confiança, importante para uma pessoa que vê sua vida afetada pelo diagnóstico de HIV. Uma noção mais ampliada de vínculo poderia comportar uma aproximação mais efetiva entre profissionais de saúde e usuários. Isso possibilitaria, a saber, conversas sobre temas como prevenção, sexualidade, uso de drogas e outros, impactando fortemente a vinculação real de um usuário a uma equipe de saúde de família. De outro lado está o receio da exposição e do estigma, que podem fazer com que alguns usuários prefiram ser acompanhados longe de onde vivem.

Isso nos faz acreditar ser necessária uma atenção especial ao vínculo real existente ou produzido entre usuário e equipe de saúde de família, bem como à 
constituição de regramentos flexíveis, sob risco de descuidar de um usuário e não garantir seu direito à saúde em virtude do modo de organização e funcionamento do serviço de saúde, configurando uma barreira.

\section{CONSIDERAÇÕES FINAIS}

As políticas dirigidas a $\mathrm{HIV} /$ Aids e APS no Brasil possuem diferentes trajetórias. Recentemente, no Brasil, tais políticas se aproximaram - inicialmente, pela incorporação de um importante papel de testagem e diagnóstico do HIV na APS (com ênfase maior em gestantes) e, posteriormente, por meio de diretrizes e experiências municipais de descentralização do cuidado a PVHA para a APS, suscitando polêmica e novos esforços. Trata-se de uma inovação social em saúde que merece ser problematizada.

Este artigo teve como objetivo contextualizar esse processo de descentralização, bem como indicar elementos configurados como tensões, desafios e possibilidades, considerando ainda o caráter recente deste movimento e a existência de poucos estudos e pesquisas. É importante destacar os desafios de ordem moral (relativos ao tema da sexualidade e ao estigma relacionado a práticas sexuais), ética (relativos ao sigilo e à confidencialidade num contexto de trabalho em equipe territorializada), técnica (manejo clínico e formação dos profissionais), organizacional interna (flexibilidade da APS para adaptar seus modos de organização, considerando necessidades e expectativas dos usuários), organizacional externa (suporte e interação de médicos de família com infectologistas da atenção especializada) e política (condução da agenda e diálogo entre diferentes atores e perspectivas). Parece-nos que o enfrentamento desses desafios pode ser positivo para PVHA, quiçá ampliando as possibilidades de acesso e qualidade do cuidado, e para a APS, que se qualificaria para este e outros desafios.

Conflitos de interesse. Nada declarado pelos autores.

Declaração. As opiniões expressas no manuscrito são de responsabilidade exclusiva dos autores e não refletem necessariamente a opinião ou política da RPSP / PAJPH ou da Organização Pan-Americana da Saúde (OPAS).

\section{REFERÊNCIAS}

1. Instituto Suramericano de Gobierno en Salud; Giovanella L, Feo O, Faria M, Tobar S, orgs. Sistemas de salud en Suramérica: desafíos para la universalidad, la integralidad y la equidad. Rio de Janeiro: ISAGS; 2012. Disponível em: http:/ /www5.ensp.fiocruz. br/biblioteca/dados/txt_403158050.pdf Acessado em agosto de 2018.

2. Miranda Junior HM. Redes de Atenção à Saúde: rumo à integralidade. Divulg Saude Debate. 2014; 52:15-37.

3. Campos GWS. Reforma política e sanitária: a sustentabilidade do SUS em questão? Cienc Saude Coletiva. 2007;12(2):301-6.

4. Starfield B. Primary care: balancing health needs, services and technology. Revised edition. Nova Iorque: Oxford University Press; 1998.

5. Victora CG, Barreto ML, do Carmo Leal M, Monteiro CA, Schmidt MI, Paim J, et al. Health conditions and health-policy innovations in Brazil: the way forward. Lancet. 2011;377(9782):2042-53.

6. Teixeira PR. Políticas públicas em Aids. Em: Parker R, org. Políticas, instituições e Aids: 1997 enfrentando a epidemia no Brasil. Rio de Janeiro: ABIA/Jorge Zahar; 1997. Pp. 43-68.

7. Galvão J. AIDS no Brasil: a agenda de construção de uma epidemia. Rio de Janeiro: ABIA; São Paulo: Ed. 34; 2000.

8. Mattos RA, Terto Jr. V, Parker R. As estratégias do Banco Mundial e a resposta à aids no Brasil. Divulg Saude Debate. 2003;27:81-94.

9. Trad LAB, Bastos ACS, Santana EM, Nunes MO. Estudo etnográfico da satisfação do usuário do Programa de Saúde da Família
(PSF) na Bahia. Cienc Saude Coletiva. 2002;7(3):581-9.

10. Zambenedetti G. O paradoxo do território e os processos de estigmatização da AIDS na atenção básica em saúde [tese de Doutorado]. Porto Alegre: Universidade Federal do Rio Grande do Sul (UFRGS): 2014. Disponível em: https://www.lume. ufrgs.br/handle/10183/115057 Acessado em agosto de 2018.

11. UNAIDS. 90-90-90: An ambitious treatment target to help end the AIDS epidemic. Joint United Nations Programme on HIV/ AIDS (UNAIDS); 2014. Disponível em: http:/ /www.unaids.org/en/resources / documents/2017/90-90-90 Acessado em agosto de 2018.

12. Brasil, Ministério da Saúde, Secretaria de Vigilância em Saúde, Departamento de Vigi lância, Prevenção e Controle das Infecções Sexualmente Transmissíveis, do HIV/Aids e das Hepatites Virais. Boletim Epidemiológico Aids/DST. Ano V, n 1. Brasília, 2017. Disponível em: http://www.aids.gov.br/ pt-br/pub/2017/boletim-epidemiologicohivaids-2017 Acessado em agosto de 2018.

13. Malta DC, Santos MAS, Stopa SR, Vieira JEB, Melo EA, Reis, AAC. Family Health Strategy Coverage in Brazil, according to the National Health Survey, 2013. Cienc Saude Coletiva. 2016;21(2):327-38

14. Brasil, Ministério da Saúde, Secretaria de Atenção à Saúde, Departamento de Atenção Básica. Política Nacional de Atenção Básica. Brasília: Ministério da Saúde; 2012. Disponível em: http://dab.saude.gov.br/portaldab/biblioteca.php?conteudo=publicacoes/ pnab Acessado em agosto de 2018.
15. Gutiérrez AC, Guerrero AVP, Cunha GT. Reflexões sobre a Atenção Básica e a Estratégia de Saúde da Família. Em: Campos GWS, Guerrero AVP, orgs. Manual de Práticas de Atenção Básica. Saúde ampliada e compartilhada. São Paulo: Hucitec; 2008. Pp. 132-254.

16. Campos GWS, Pereira Junior N. Primary care in Brazil, and the Mais Médicos (More Doctors) Program in the Unified Health System: achievements and limits. Cienc Saude Coletiva. 2016;21(9): 2655-63.

17. Melo EA. Where now for the Mais Médicos (More Doctors) Program? Cienc Saude Coletiva. 2016;21(9):2672-4.

18. Brasil, Ministério da Saúde, Secretaria de Vigilância em Saúde, Departamento de DST, Aids e Hepatites Virais. Caderno de Boas Práticas em HIV/AIDS na Atenção Básica. Brasília: Ministério da Saúde; 2014. Disponível em: http://www.aids.gov.br/ pt-br/pub/2014/caderno-de-boas-praticas-em-hivaids-na-atencao-basica Acessado em agosto de 2018.

19. Bonet O. Os médicos da pessoa: um olhar antropológico sobre a medicina de família no Brasil e na Argentina. Rio de Janeiro: 7Letras; 2014.

Manuscrito recebido em 24 de dezembro de 2017 Aceito em versão revisada em 13 de agosto de 2018. 
ABSTRACT In Brazil, the Unified Health System (SUS) consists of a hierarchical and regionalized network of health services coordinated from the primary health care (PHC) level, which is also the entry point into the system. Recently, as a result of

HIV/AIDS management at the primary care level in Brazil: a challenge for the Unified Health System?

Keywords Primary health care; acquired immunodeficiency syndrome; comprehensive health care; Brazil. new guidelines and experiences in Brazil, $\mathrm{PCH}$ has been assigned a more substantial role in the care and management of people living with HIV/AIDS, tasks traditionally performed at specialized clinics. The present article contextualizes and explores the problems involved in this recent process of providing decentralized care to people living with HIV/AIDS in the context of the SUS. Since 2011, new diagnostic technologies (such as rapid testing) have become available at PHC units in Brazil, expanding access to testing and leading to an increase in the number of HIV diagnoses performed at the PHC level. Since 2013, new guidelines and recommendations have also supported care for people with HIV/AIDS in PHC units. The present article examines the relationship between PHC and specialized care, issues of access, stigma, and confidentiality in PHC, and the mode of organization and functioning of family health teams, especially the formal link between residents in catchment areas and health teams and workers. In conclusion, many challenges-moral, ethical, technical, organizational, and political-have to be faced in order to increase access and quality of care in the context of PHC for people living with HIV/AIDS in Brazil.
RESUMEN

\section{Cuidados de pacientes con $\mathrm{VIH} /$ sida y atención primaria en Brasil: desafíos para la atención en el Sistema Único de Salud?}

Palabras clave

En Brasil, el Sistema Único de Salud (SUS) prevé la organización de una red jerarquizada y regionalizada de servicios de salud, teniendo la atención primaria en salud (APS) como ordenadora y puerta de entrada para los servicios. Recientemente, nuevas directrices y experiencias brasileñas otorgaron a la APS un papel de mayor protagonismo en el tema de las políticas de VIH/sida, que hasta entonces desarrollaban su componente asistencial centralizado en servicios especializados. Este artículo contextualiza y explora los problemas de este reciente proceso de descentralización del cuidado a las personas que viven con VIH/sida en el SUS. A partir de 2011, nuevas tecnologías diagnósticas (como las pruebas rápidas) fueron implantadas en la APS en Brasil, ampliando el acceso a la prueba y promoviendo un aumento del número de diagnósticos de VIH en la APS. A partir de 2013, las directrices y recomendaciones incentivaron también el seguimiento de las personas con $\mathrm{VIH} /$ sida en el marco de la APS. En este contexto, el presente artículo examina la relación entre la APS y atención especializada, los temas de acceso, estigma y confidencialidad en la APS y el modo de organización y funcionamiento de los equipos de salud de la familia, así como la vinculación formal entre los habitantes y los equipos de APS. Se concluye que es necesario enfrentar varios desafíos (de orden moral, ético, técnico, organización y política) para ampliar las posibilidades de acceso y la calidad del cuidado en la APS para las personas que viven con VIH/ sida en Brasil.

Atención primaria de salud; síndrome de inmunodeficiencia adquirida; atención integral de salud; Brasil. 